\title{
Revision rhinoplasty using autologous rib cartilage in Asians
}

\author{
Tae-Bin Won', Hong-Ryul Jin² \\ 'Department of Otorhinolaryngology-Head and Neck Surgery, Seoul National University Bundang Hospital, Seongnam 13620, South Korea. \\ ${ }^{2}$ Dr Jin's Premium Nose Clinic, Seoul 06524, South Korea.
}

Correspondence to: Dr. Hong-Rul Jin, Dr. Jin's Premium Nose Clinic, Seoul 06524, South Korea. E-mail: doctorjin@daum.net

How to cite this article: Won TB, Jin HR. Revision rhinoplasty using autologous rib cartilage in Asians. Plast Aesthet Res 2019;6:6. http:// dx.doi.org/10.20517/2347-9264.2018.82

Received: 17 Dec 2018 First Decision: 13 Jan 2019 Revised: 31 Jan 2019 Accepted: 12 Feb 2019 Published: 22 Mar 2019

Science Editors: Hong Ryul Jin, Raúl González-García Copy Editor: Cai-Hong Wang Production Editor: Huan-Liang Wu

\begin{abstract}
A considerable part of revision rhinoplasty in Asians is associated with problems arising from the use of alloplastic implants. Revising alloplast associated problems of the nose mostly requires the use pf autogenous grafting material to minimize complications and maximize favorable outcomes. Although remnant septal cartilage and/or conchal cartilage can be used, as the deformity becomes more severe, adequate revision requires more volume and strength of grafting materials. Autogenous rib cartilage may be the most practical choice in these circumstances. In this review, common causes of revision rhinoplasty in Asians are discussed together with operative techniques with emphasis on the use of autologous rib cartilage.
\end{abstract}

Keywords: Rhinoplasty, Asian, revision, rib cartilage

\section{INTRODUCTION}

The increasing number of primary rhinoplasties coupled with heightened patient expectations, has led to an increase of revision surgery. In a recent study of revision rhinoplasty in Asians, we have shown that most revision rhinoplasties were associated with problems arising from the use of alloplastic implants, reflecting the trend of using alloplastic implants in many Asian countries ${ }^{[1]}$. Although studies and years of clinical experience have proven that these implants can be used safely, incorrect surgical techniques coupled with inappropriate patient selection, can cause an array of complications.

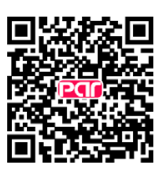


Table 1. Causes of revision rhinoplasty in Asians ${ }^{[1]}$

\begin{tabular}{ll}
\hline Main etiology for revision rhinoplasty $(\boldsymbol{n}=\mathbf{5 2})$ & \\
\hline Alloplast related $(n=33)$ & $33(63 \%)$ \\
Deviation & 12 \\
Foreign body & 5 \\
Extrusion & 5 \\
Infection & 4 \\
Unnatural look & 4 \\
Contracture (short nose) & 3 \\
Alloplast unrelated $(n=19)$ & $19(37 \%)$ \\
Mainly upper two-thirds problem $(n=12)$ & \\
Residual deviation & 7 \\
Dorsal irregularity or depression & 4 \\
Residual hump & 1 \\
Mainly tip problem ( $n=7)$ & \\
Tip underprojection $($ loss of projection) & 2 \\
Upturned, overrotated tip & 2 \\
Visible graft & 2 \\
Tip deviation & 1 \\
Nasal obstruction & 4 \\
\hline
\end{tabular}

Like other revision surgeries, the difficulty of revision rhinoplasty arises not only from the effort it takes to correct the specific deformities and scars left by the primary surgery, but also from the psychological stress experienced by both the patient and the surgeon. An in-depth understanding and sufficient experience in various rhinoplasty techniques together with familiarity with the alloplasts' characteristics and related complications become necessary with dealing with the highly variable cases of Asian revision rhinoplasty ${ }^{[2-7]}$.

Remnant septal cartilage and/or conchal cartilage can be used for the revision procedure. However, as the deformities become more severe, adequate revision requires more volume and strength of grafting materials. This usually far exceeds available septal cartilage and the need for adequate strength precludes the use of conchal cartilage. Autogenous costal cartilage is a favorable grafting material and may be the only practical choice in these circumstances.

In this review, common causes of revision rhinoplasty in Asians are discussed as well as operative techniques that focus on the use of autologous costal cartilage are presented.

\section{REVISION RHINOPLASTY IN ASIANS}

The main reasons for revision rhinoplasty in Asians often involve issues with alloplastic implants. Common indications for revision rhinoplasty that we have encountered are as follows and are summarized in Table $1^{[1]}$.

Alloplast-related complications such as deviation, extrusion, infection, short and contracted nose after multiple surgeries involving alloplastic impants, dorsal deviation and/or irregularity and tip problems related to septal extension graft.

\section{Alloplast related complications}

Even though trends change silastic implants remain the single most commonly used alloplastic implant in Asia ${ }^{[7-9]}$. Despite the fact that they can be better tolerated by the thicker skin and soft tissue envelope (SSTE) of the Asian nose ${ }^{[10]}$, silicone implants have been heavily criticized for their association with various complications ${ }^{[11]}$. Typical examples of alloplast-related complications include unnatural or operated appearance, deviation, extrusion of the implant, infection, foreign body reaction and compromised SSTE. Proper selection of patients, adherence to proper surgical techniques and acquiring the necessary techniques to manage complications when they occur are important ${ }^{[1,7]}$. 


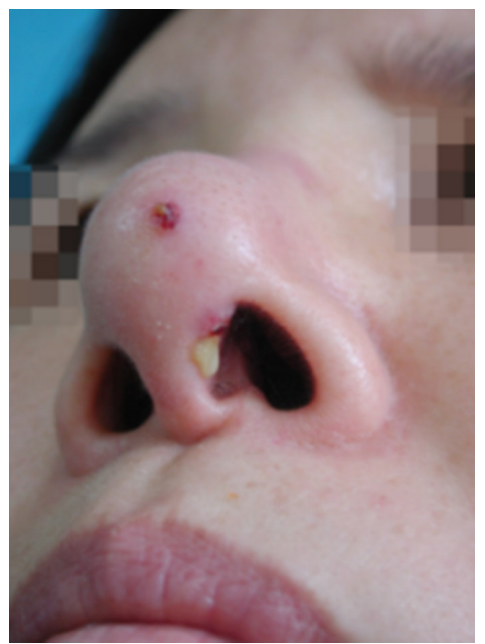

Figure 1. Infection of an alloplastic implant used for dorsal augmentation showing pus coming out from the nasal tip

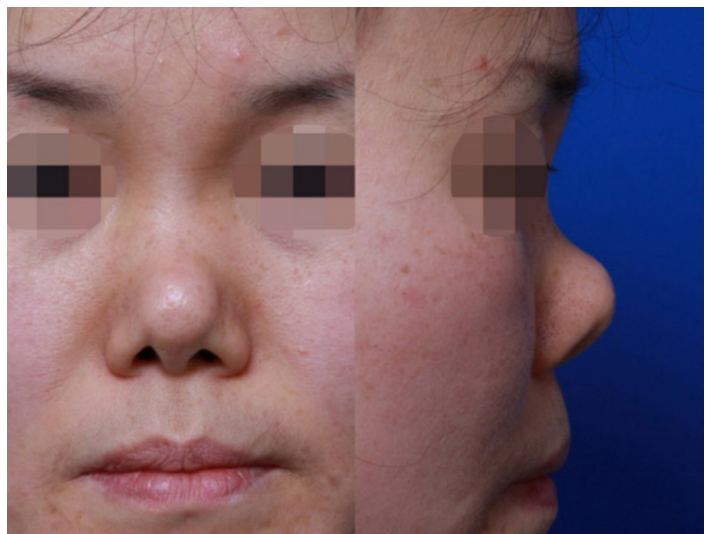

Figure 2. Short and contracted nose after multiple rhinoplasties

Infection with alloplastic implants can occur immediately or years after surgery [Figure 1] ${ }^{[12-15]}$. Although aggressive antibiotic therapy can be undertaken, the chances of implant salvage are low, especially in cases where e-PTFE has been used ${ }^{[13,15]}$. There is no consensus on the timing of the definitive revision rhinoplasty after implant removal. Currently, the mainstay of treatment is a staged approach with removal of the alloplast and subsequent revision operation after infection control. Although it can provide a more sterile environment, the delay in surgery can result in contracture of the overlying SSTE not to mention the added frustration of the patient ${ }^{[16]}$. In recent years, we have performed many reconstruction using autologous cartilage, especially rib cartilage, after removal of an infected alloplast. We have found that the result is favorable with minimal chances of infection and resorption ${ }^{[17]}$.

\section{Short, contracted nose}

A short, contracted nose is a devastating complication usually associated with repeated surgery using alloplastic grafting material ${ }^{[17-19]}$. The distorted anatomy lies not only in the structural support but also in the overlying SSTE. The exact pathogenesis is yet unknown but possible etiologies include capsular contraction around the implant, lower lateral cartilage necrosis by long term pressure from implants and chronic inflammation that eventually leads to progressive scar contracture ${ }^{[20]}$. As the contracture progresses, the so-called snub nose deformity develops [Figure 2]. Therefore, caudal rotation of the tip together with superior movement of the nasion to elongate the nose are necessary. Building a firm foundation with autologous grafting material that can counteract the contractile forces of the skin is the key and rib 

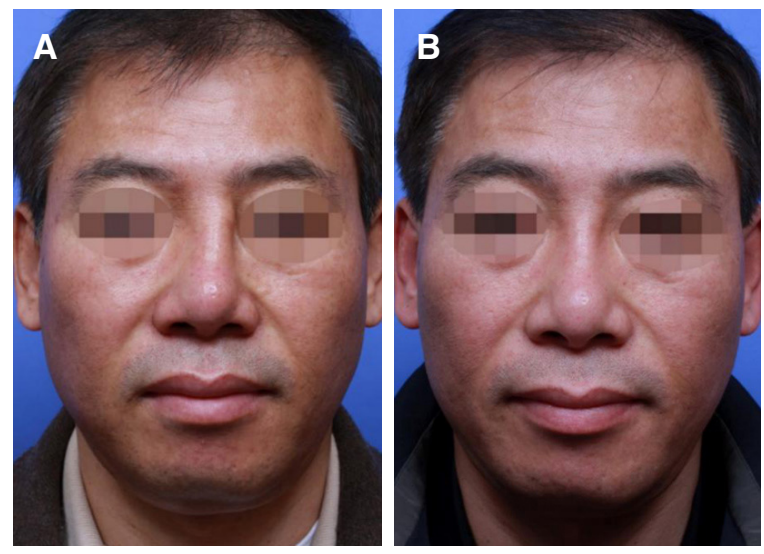

Figure 3. Pre and postoperative 1 year frontal photograph of a 52-year-old male patient with deviation of the silicone implant (A, B). The implant was removed together with the surrounding capsule and dorsal augmentation was performed using autogenous rib cartilage. Tip projection and rotation was achieved using a septal extension graft

cartilage is often used to fulfill this purpose. Often conchal composite grafts are needed to correct the deficient vestibular mucosa. A non-alloplast dorsal onlay graft that fills the dorsal defect after removal of the previous alloplast is also preferred.

\section{Dorsal deviation/irregularity}

Residual dorsal deviation is most often caused by failure to recognize or correct the pre-existing deviation. Improper osteotomies with or without adequate correction of the septum is the main cause. Deviation of the dorsal graft/implant and warping of the costal cartilage graft can be other reasons. Complete realignment employing restorative measures to straighten the bone and cartilaginous structures are required. If residual deviation persists after adequate structural realignment, camouflage grafts need to be applied [Figure 3].

A supratip depression or fullness after dorsal augmentation is not uncommon. Careful design of the implant and fine adjustment with additional grafts at the supratip is often necessary during primary rhinoplasty. Radix irregularity is more common when the radix is augmented with cartilage. To avoid this, the radix graft should be morcelized and inserted under a layer of soft tissue. Mastoid periosteum provides a good grafting source to smoothly elevate the radix area.

\section{Tip problems related with septal extension graft}

The recent trend of using septal extension graft for tip surgery in Asian rhinoplasty has created an array of complications such as overly aggressive tip projection (Pinocchio nose), deviated, asymmetric tip, pain and nasal obstruction. Aggressive tip projection using septal bone or Medpor is a common reason for tip pain and tenderness [Figure 4]. Removing stiff materials and restoring adequate projection with autologous cartilage is the best solution in these patients.

Inadequate midline stabilization of the septal extension graft is a common reason for tip deviation, nostril asymmetry, and nasal obstruction due to caudal septal deviation. This can often be avoided by securely suturing the graft to the anterior nasal spine and positioning the end into the midline in the overlapping type of septal extension graft. In the end to end type septal extension graft, reinforcement can be achieved with extended spreader grafts.

\section{GRAFTING MATERIAL IN REVISION RHINOPLASTY}

Revision rhinoplasty requires a large quantity of implants for grafting, supporting, and reconstruction. We prefer autologous cartilage for revision rhinoplasty in order to avoid further infection and soft 

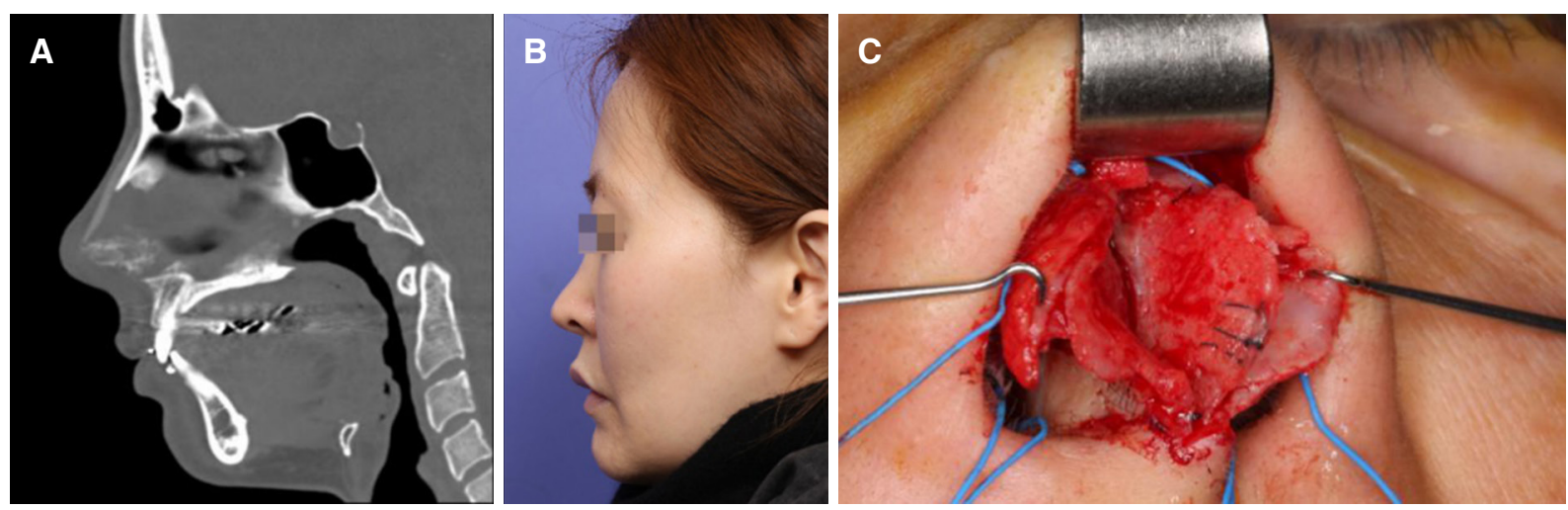

Figure 4. Sagittal CT scan of a 36-year-old female patient who underwent a rhinoplasty 6 years ago complaining of nasal tip pain, tenderness, and overly projected tip. Dorsal silicone implant and suspicious bone at the caudal septal area used as a septal extension graft (A, B); Intraopertaive photos show an L-shaped Medpor implant used as septal extension graft (C). After implant removal and reconstructing of the tip without over projection using autologous cartilage, the pain disappeared

tissue contraction. Remnant septal and ear cartilage are the first choice of grafting materials in revision rhinoplasty cases with minor deformities. However, secondary rhinoplasty more often than not requires a larger amount of tissue that requires a robust source of grafting material. This usually exceeds the available septal cartilage and the need for adequate strength precludes the use of conchal cartilage. Autogenous costal cartilage may be the only practical choice in these circumstances. Common scenarios that frequently require the use of rib cartilage include the following: contracted short nose, significant loss of dorsal volume and/or septal support and/or tip support that is usually associated with removal of the alloplast.

We rarely use homologous rib cartilage because we believe that it is unpredictable in terms of long-term resorption. In cases with problems of the skin-soft tissue envelope, temporalis fascia, costal perichondrium, mastoid periosteum, or autologous dermis is used to reinforce the skin that may have been overly-thinned or weakened. Homologous fascia or dermis (Alloderm ${ }^{\circ}$, Surederm ${ }^{\circ}$ ) can be feasible alternatives. Lastly, although not common in our hands, alloplastic implants can be used again for revision, if the patient recognizes and agrees to the risks of complication, when there is no demonstrable infection, and in the presence of relatively thick skin.

\section{COSTAL CARTILAGE HARVESTING}

Before harvesting, it is prudent to check the rib series X-ray to look for possible calcifications. Even young patients can have severe calcification of the costal cartilage, which is more common in females. Calcification makes harvesting and carving of the cartilage more difficult and if totally calcified, it cannot be used as a grafting material.

The costal cartilage is commonly harvested from the sixth or the seventh rib. The incision is made directly over the chosen rib in male patients and just above the infra-mammary crease in female patients to conceal the chest scar [Figure 5]. The size of the incision may vary and is usually $2 \mathrm{~cm}$ in length in thin skinned patient and $2-2.5 \mathrm{~cm}$ in the thick skinned patient. The costochondral junction is confirmed by serial puncture with a 26-gauge needle for precise placement of the incision. The skin and subcutaneous tissue are incised with a no.10 blade and the subcutaneous tissue is retracted using retractors until exposing the external oblique muscles. Instead of cutting them with a Bovie, the muscle fibers are separated with Kelly forceps and retracted with an Army-Navy retractor which can minimize postoperative pain. After adequate exposure, two parallel incisions are made along the superior and inferior borders of the rib cartilage, leaving an intact central strip of perichondrium on the anterior surface. Several small incisions are made perpendicular to the longitudinal incision to facilitate reflection of the perichondrium [Figure 6]. 

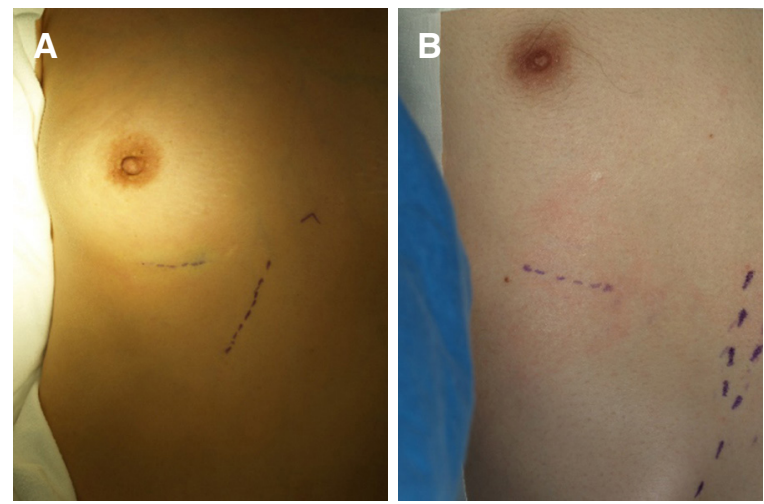

Figure 5. Incision for costal cartilage harvest in women (A) and men (B)
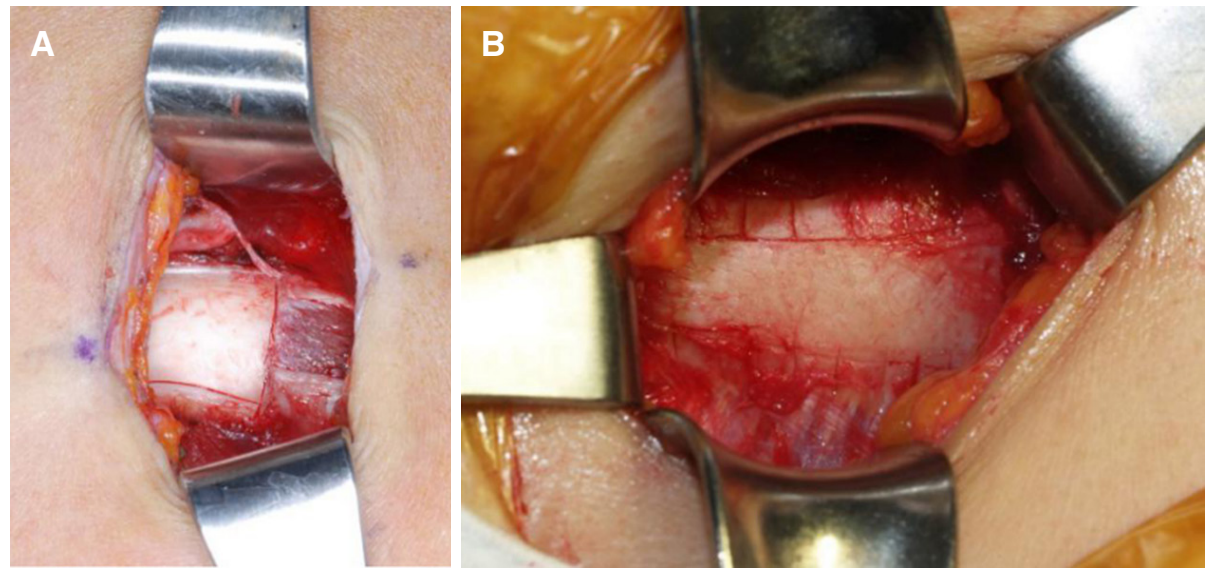

Figure 6. Bone cartilage junction (A); multiple small perichondrial incisions are made perpendicular to the longitudinal one to facilitate circumferential reflection of the perichondrium (B)

Harvesting can be facilitated by performing a medial cut before completing the dissection of the posterior surface of the costal cartilage. The initial incision is made with a blade and the cartilage cut is completed with a Freer elevator to avoid inadvertent pleural injury. After the lateral cut, small two-prong retractors are used to pull the costal cartilage exposing the posterior surface. The perichondrium of the posterior surface is dissected with a curved elevator and delivered. Typically, a 3-4 cm length of costal cartilage can be harvested together with the central strip of perichondrium [Figure 7]. When necessary, the cartilaginous cut can be extended up to the synchondrosis portion to obtain a longer graft.

Testing for air leakage is performed by filling the dissection pocket with saline and performing positivepressure hyperventilation. If no air leakage is confirmed, the donor site is packed with antibiotic-soaked gauze until the end of the operation. Extra cartilage can be harvested during the operation or remnant cartilage can be reinserted for future use. If air leakage is noted, a nelaton catheter is inserted at the leakage site and repaired in a purse-string manner. The nelaton catheter is removed while exerting positivepressure ventilation. The separated muscles are approximated to diminish postoperative pain and the wound is closed layer by layer using 4-0 vicryl. A drain is usually not necessary. If the skin margins are macerated, they should be trimmed before suturing with a 6-0 nylon which is removed on the seventh to tenth postoperative day. A routine chest X-ray to check for pneumothorax is not mandatory if the surgeon is confident that there was no pleural injury. However it should be performed if the patient develops chest signs and symptoms. Rarely, pneumothorax can occur even though leakage was not evident during surgery, in which case, a chest tube is inserted to expand the collapsed lung. 


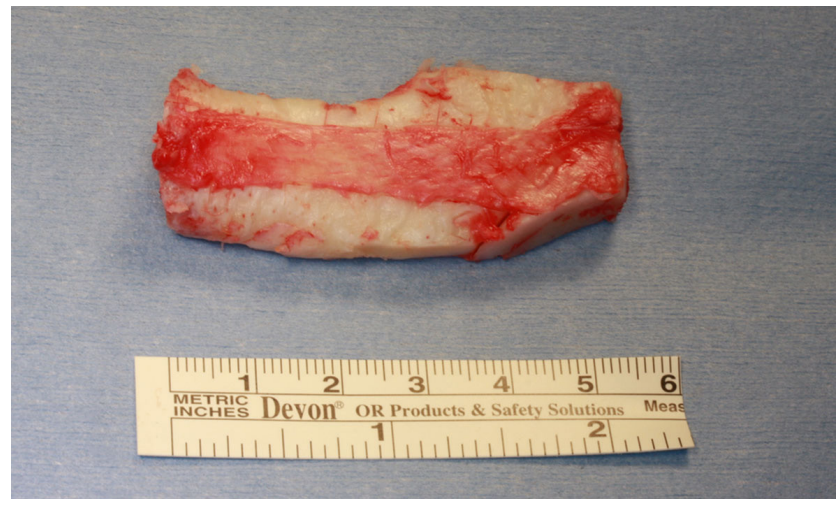

Figure 7. Harvested costal cartilage together with the central strip of perichondrium

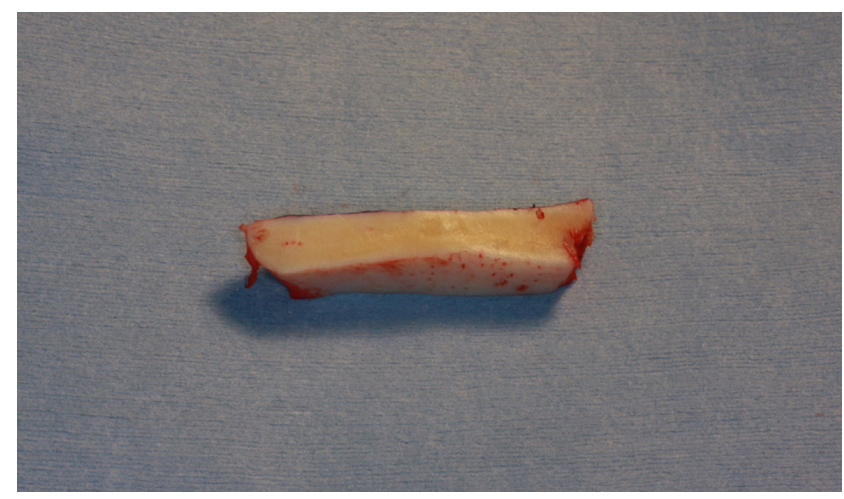

Figure 8. Section through the costal cartilage reveals a yellowish core region and a whitish peripheral region

\section{TAILORED USE OF COSTAL CARTILAGE}

Harvested autologous costal cartilage is designed into various shapes and sizes depending on the purpose of the graft.

\section{Costal cartilage for dorsal implant}

There are various ways of using the costal cartilage for dorsal augmentation, these include, dorsal augmentation as a single piece, stacked layered pieces, and diced cartilage wrapped with fascia.

\section{Dorsal augmentation in one piece}

Costal cartilage consists of a central core and a peripheral region surrounding the core [Figure 8]. A balance within the cartilage is maintained by the internal stress created by the two competing regions. The peripherally cut cartilage warps more than the centrally cut segment ${ }^{[21,22]}$. Side-to-side warping is clinically more evident due to diminished soft tissue resistance in this dimension [Figure 9].

To design an implant for dorsal augmentation, the periphery is symmetrically cut away, leaving a central core. The central part is carved with a No.10 blade. The cartilage is periodically soaked in saline for 1020 min to carefully monitor signs of warping. Once warping is evident, the remaining peripheral concave portion of the cartilage is cut out keeping the central core. Making additional cuts in the graft can further minimize warping. The final dorsal graft is a canoe-shaped graft when seen from above. When seen from the lateral view, it has a slightly concave side that comes into contact with the nasal dorsum, and the skin side is slightly convex [Figure 10]. Perichondrium placement on the undersurface of the radix portion and suture fixation of the graft to the upper lateral cartilage can reduce movability and decrease the chances 


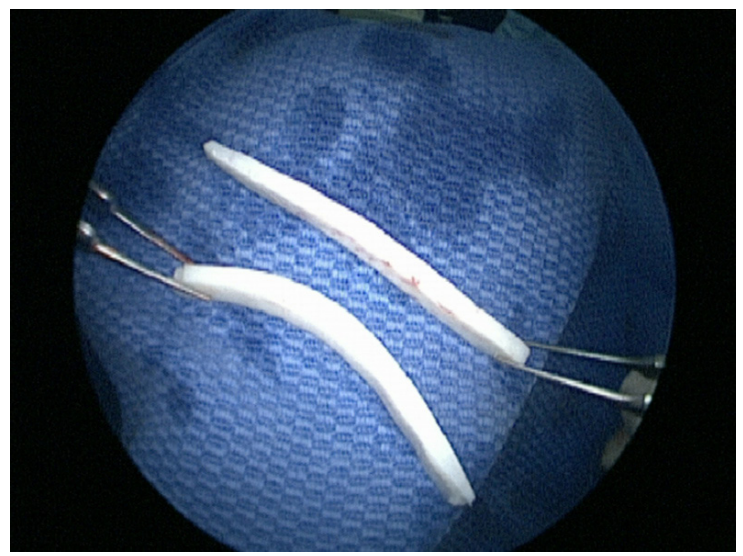

Figure 9. Warping of the costal cartilage
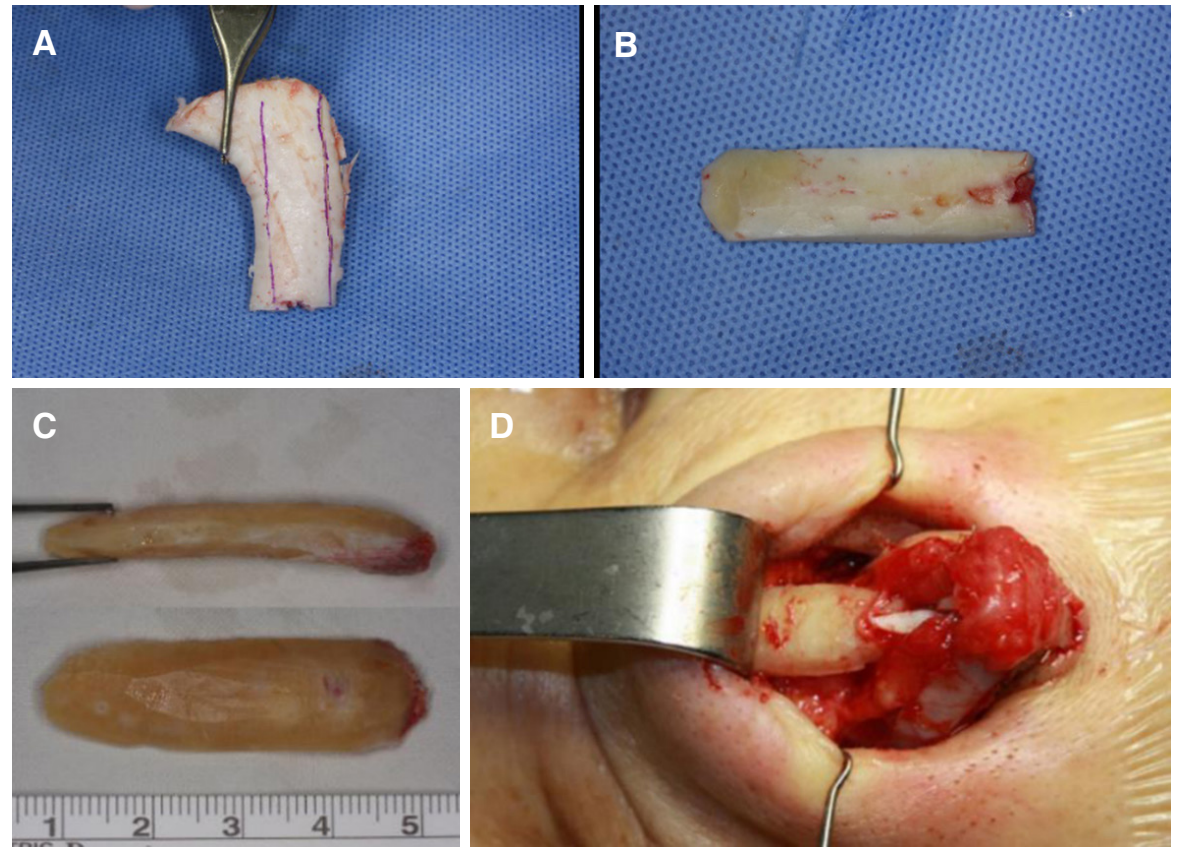

Figure 10. A dorsal onlay graft carved from a rib cartilage. A straight portion of the harvested rib cartilage is selected (A); the peripheral portion is excised leaving a central portion for additional carving (B); the final graft has a canoe-like shape from the frontal view and a slightly convexity in the dorsal side when seen from the lateral view (C); insertion of the dorsal graft (D)

of migration [Figure 11]. When wide dissection of the dorsal skin is necessary, fixation of the carved rib cartilage needs additional procedures. A K-wire fixation for two weeks or transcutaneous suture fixation of the graft to the nasal bony pyramid helps to stabilize the graft [Figure 12].

\section{Dorsal augmentation with layered costal cartilage}

Alternatively, the costal cartilage can be cut in layers or long strips which can be stacked to be used as the dorsal graft. Usually strips of $1.0-1.5 \mathrm{~cm}$ by $3-4 \mathrm{~cm}$ with $1 \mathrm{~mm}$ thickness are used. Obtaining strips with consistent thickness is not easy. Using a dermatome blade is helpful to achieve this goal [Figure 13]. The number of strips to stack depends on the desired dorsal height and are sutured with 5-0 PDS or nylon. More often than not, the strips bend or warp. By stacking the warped strips in a way that can counteract the forces of one another can succeed in obtaining a straight dorsal graft. Special care is taken to bevel all the edges. Fascia or perichondrium is used to cover and camouflage the graft. 


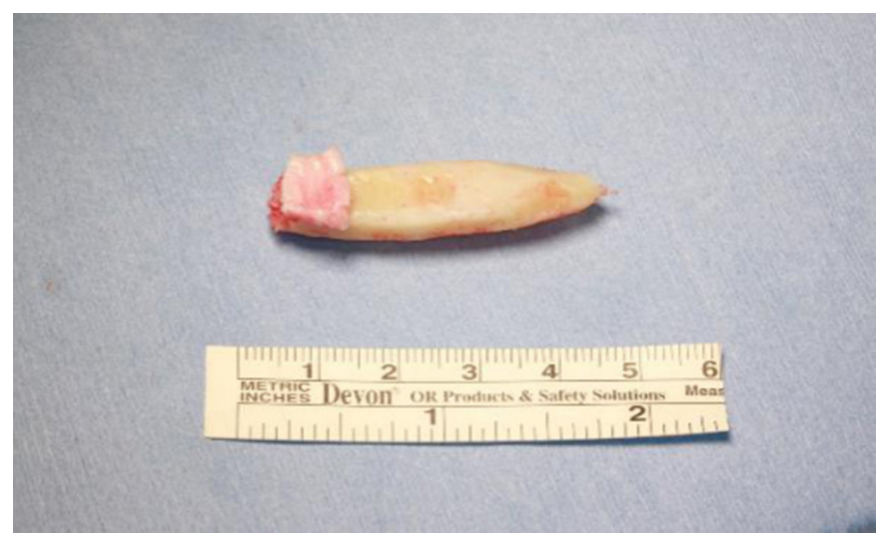

Figure 11. Perichondrium on the undersurface of the radix portion of the graft with slight rasping of the bone can reduce mobility and decrease the chances of migration

\section{Dorsal augmentation with diced costal cartilage wrapped in fascia}

The use of diced cartilage for dorsal augmentation has been previously published. Although similar in concept, there have been differences in the source of cartilage and the way they are prepared before insertion. The most common source of cartilage is the septum and ear if the needed volume is not small. Superiority of one versus another has not been clearly shown. The diced cartilage is usually wrapped before being inserted into the dorsal pocket so that it can conform to the desired shape and height of the dorsum. There are also a wide range of materials to wrap the diced cartilage in, with temporalis fascia being the most popular [Figure 14]. To avoid dorsal irregularities, costal cartilage is diced into fine pieces (usually 0.5-1 mm). Wrapping them with thicker fascia lata also helps to avoid palpable protuberances. The radix and supratip portions need extra attention in order to avoid slight depression in the long-term follow up.

\section{Costal cartilage for structural support: SEG and extended spreader grafts}

For structural grafts, the costal cartilage is designed into flat, straight pieces of thin cartilage. To minimize warping, the cartilage is cut longitudinally or tangentially, leaving symmetric peripheral portions on both sides of the central core portion. However, when warping occurs, affected fragments can be split in half and used as bilateral extended spreader grafts, compensating for the curvature [Figure 15]. If a thin straight piece of septal cartilage remains, it can be used as septal extension graft and costal cartilage can be used as extended spreader grafts to fix it.

\section{Costal cartilage used for tip modifications}

Examples are lateral crural strut grafts, lateral crural onlay grafts and tip onlay grafts. Usually thin, beveled slices of costal cartilage are carved with a 10 blade [Figure 16]. Grafts should be symmetrical when applied bilaterally and not thick in thin skinned patients. Cap graft can also be fashioned from the rib cartilage. The authors prefer an elliptical shaped, well beveled cartilage graft for the cap graft. The perichondrium can be draped over the cap graft for smooth transition from the dome to the soft tissue triangle.

\section{Use of rib cartilage perichondrium}

The rib perichondrium is a valuable grafting material in revision rhinoplasty. Usually the anterior perichondrium of the harvested rib cartilage is used but additional perichondrium can be harvested from the adjacent ribs (superior or inferior). Applications include the following: placement in the undersurface of the dorsal graft to increase friction and avoid mobility of the dorsal graft, on the dorsum for a radix graft or to camouflage any dorsal irregularities, on the tip to conceal graft edges and in any other areas of thinned/ damaged skin. When harvesting the rib, the authors prefer harvesting periosteum of the rib bone near the costochondral junction together with the perichondrium. Periosteum is thicker than perichondrium and helps to camouflage more especially when used at the radix portion. 


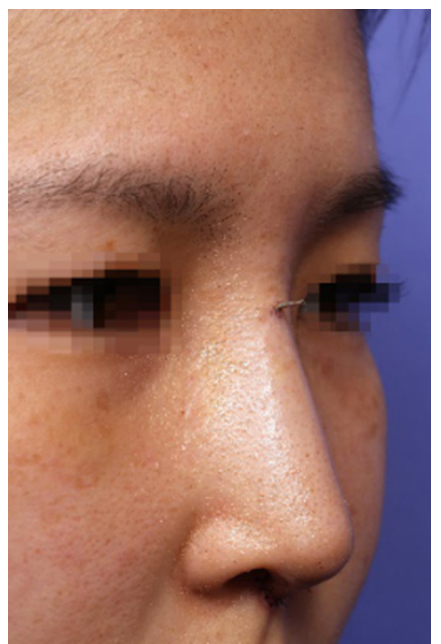

Figure 12. Fixation of the dorsal graft with K-wire

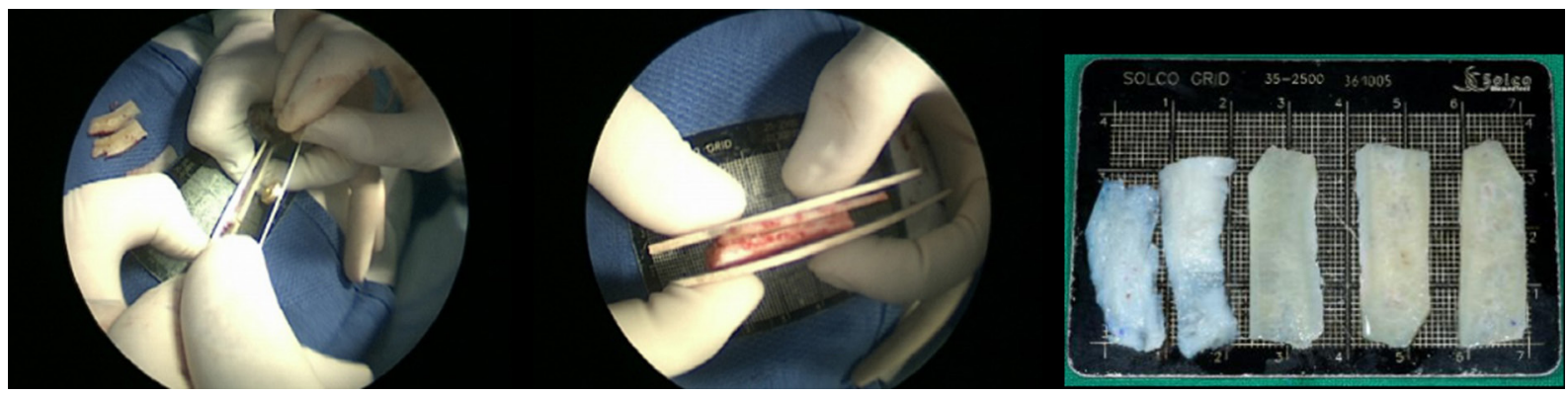

Figure 13. Layers of costal cartilage cut with a dermatome blade can be stacked for dorsal augmentation
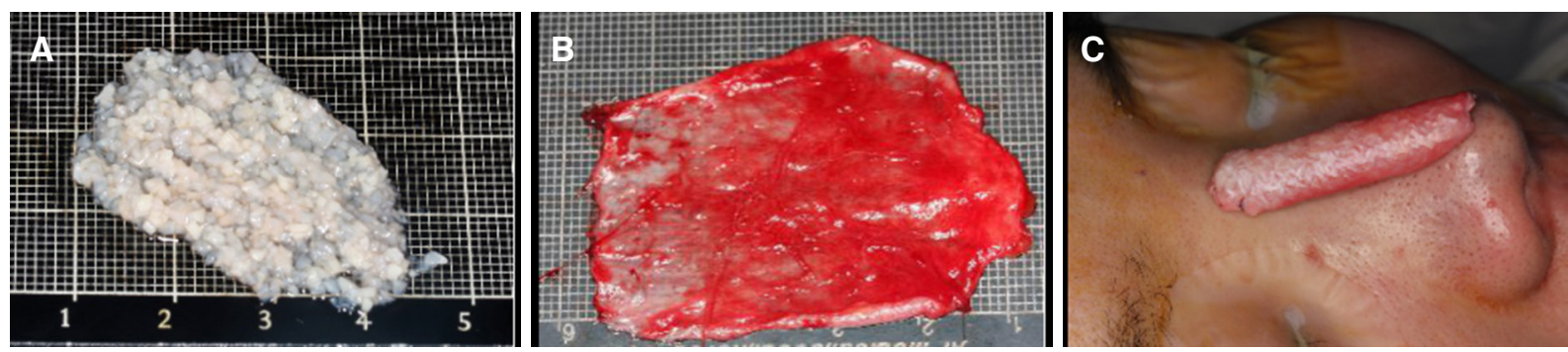

Figure 14. Dorsal augmentation with diced costal cartilage (A) wrapped in temporalis fascia (B); Final dorsal implant before insertion (C)

\section{COMPLICATIONS OF RIB CARTILAGE AND ITS MANAGEMENT}

Complications associated with using rib cartilage in rhinoplasty include warping, migration, infection, operated look and donor site morbidity such as pain, pneumothorax, and chest scar. We hereby introduce ways to deal with some of them.

Even with every preventive measure such as using the core of the rib cartilage, balanced carving, repeated immersion and checking for warping, creation of a tight pocket for insertion, and suture fixation on the dorsum, warping of the dorsal graft can occur [Figure 17]. When warping occurs postoperatively, we take out the curved rib graft and reinsert it after carving it again into a straighter piece which is possible in most instances. If that is not possible, we dice the rib graft and insert it after wrapping in temporalis fascia. In our experience, autogenous rib cartilage is able to maintain its original volume years after surgery. If 

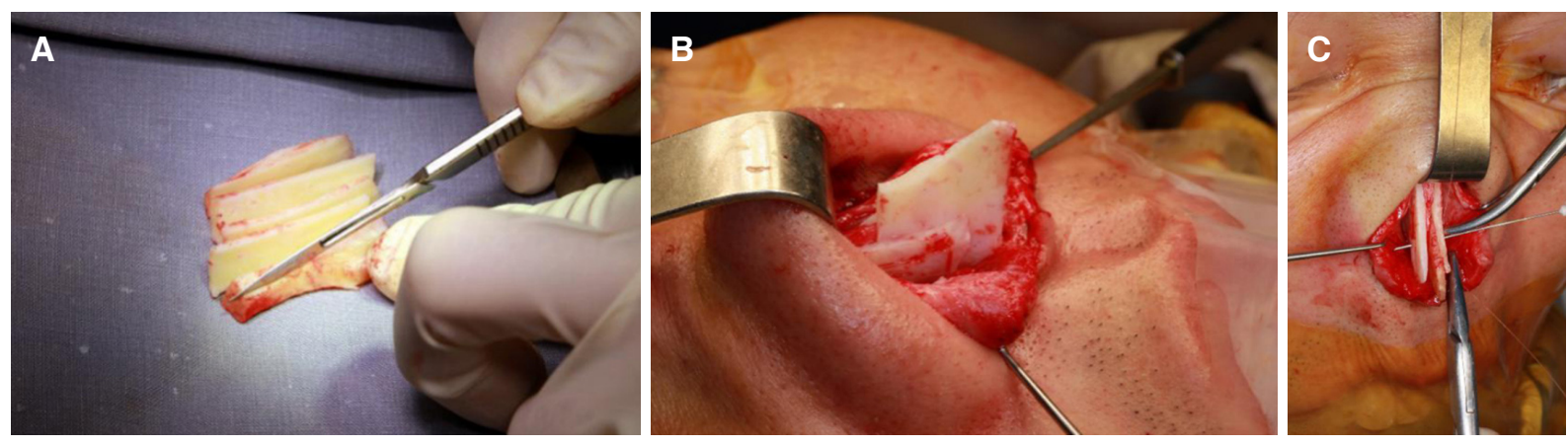

Figure 15. Costal cartilage is cut in straight layered strips(A); septal extension graft with bilateral extended spreader grafts carved from layers of costal cartilage $(B, C)$

slight volume loss occurs while taking out or recarving the rib cartilage, adding some soft tissue such as the mastoid periosteum or temporalis fascia may help in keeping the original volume or camouflaging the junction area such as radix or supratip.

Although not frequent, infection can occur after use of autologous rib cartilage. The use of autogenous material is not completely without the risk of infection, especially when rib cartilage is used for multiply revised cases. With the use of IV antibiotics, adequate drainage and proper local dressing, the costal cartilage graft can be salvaged without the need of removal. When managing the infection, early detection and aggressive intervention are important. With delayed detection and timid intervention, infection cannot be controlled completely and may end up with complete debridement of the already infected, resorbed, fragmented rib cartilage ${ }^{[16]}$.

Migration of the costal cartilage graft is rare. Possible reasons include, an excessive wide pocket around the radix area, inappropriate fixation of the costal cartilage and remnant capsule. To prevent migration, complete removal of the underlying capsule, roughening of the radix with rasps, and placement of perichondrium on the undersurface of the carved graft at the radix area are techniques to consider. Occasionally, a K-wire fixation of the graft to the underlying nasal bone at the radix can be performed.

To avoid the unnatural operated look, the authors do the following: (1) avoid over-augmentation, especially at the radix area and try to set the starting point of the dorsum to the interpupillary line; (2) fill the radix with soft tissue such as perichondrium to avoid an interrupted look from the forehead to the nose; (3) when augmenting the dorsum, narrowing the bony base with osteotomies is avoided; (4) the width of dorsal graft is kept adequately wide and the edges are beveled/carved so that transition from the sidewall to the dorsum is smooth; (5) the dorsal graft is covered with soft tissue such as temporalis fascia or mastoid periosteum especially when the skin is thin or damaged.

\section{REVISION RHINOPLASTY CASES WITH COSTAL CARTILAGE}

\section{Case 1: contracted nose after previous rhinoplasty}

A 28-year-old male present with short nose and nasal obstruction. He had only had one previous rhinoplasty using silicone implant and it was removed due to infection. He had a typical post-surgical short, contracted nose showing exaggerated nostril show in the frontal view and severely cephalically rotated nasal tip with low-set nasion in the lateral view [Figure 18A-C].

The first step of his surgical procedure consisted of a wide dissection of the skin-soft tissue envelope. The silicone capsule and thick scars were excised and the lower lateral cartilage was released from the upper lateral cartilage and pyriform aperture. 

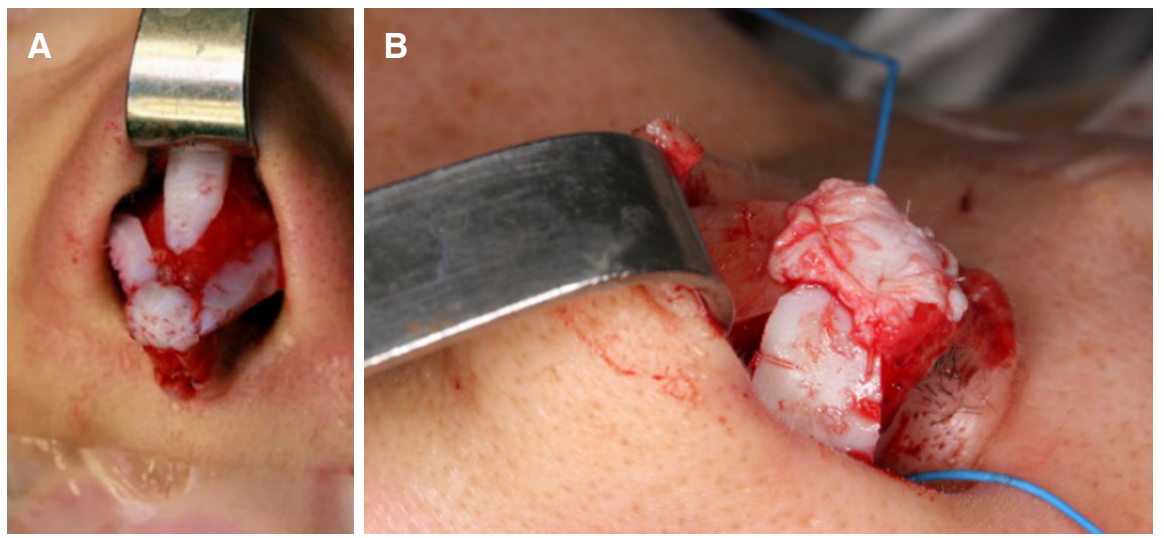

Figure 16. Costal cartilage used for tip-plasty. A dorsal graft, cap graft and bilateral lateral crural onlay grafts are shown (A); perichondrium covered over the cap graft can conceal irregularities (B)
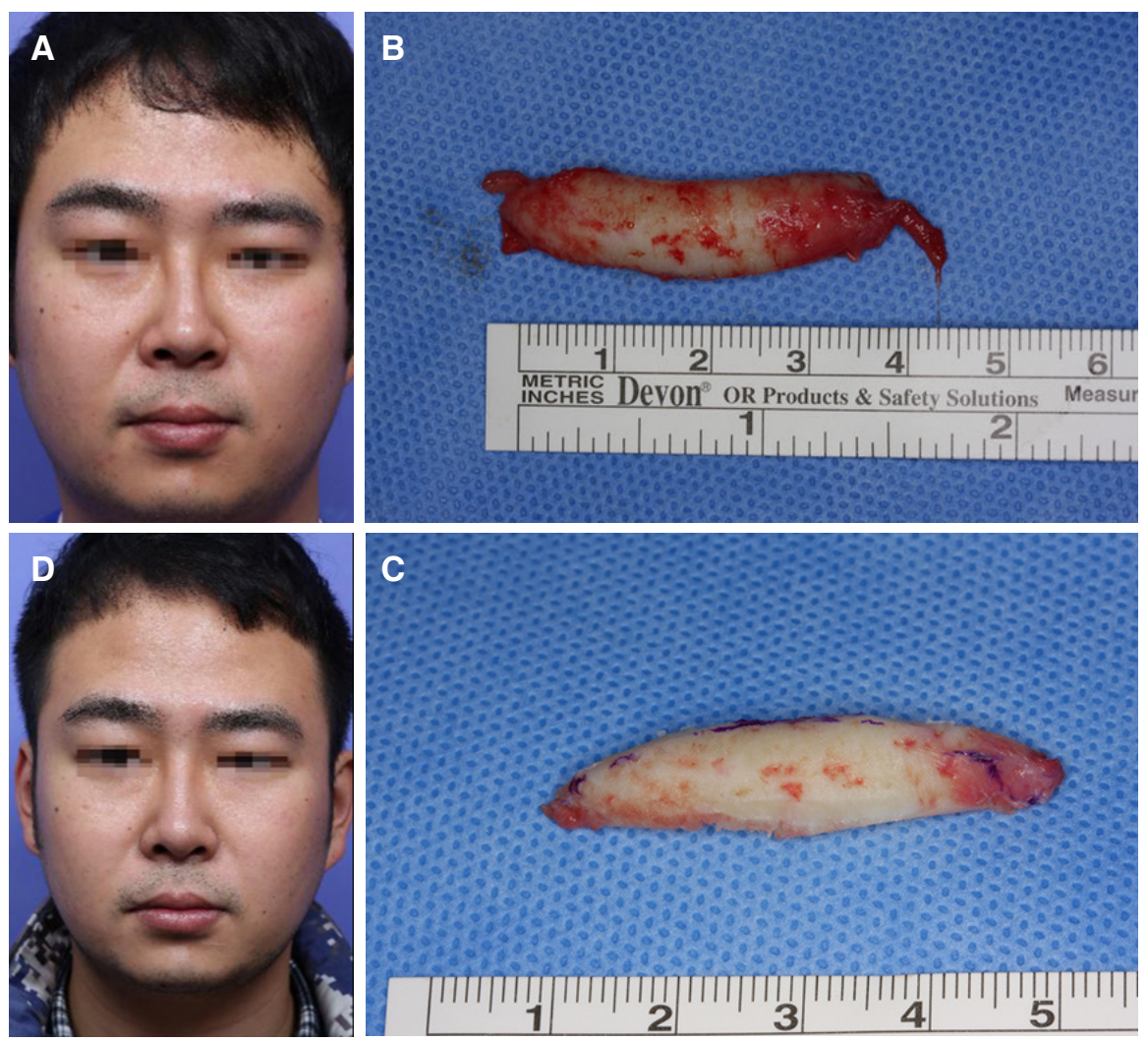

Figure 17. Warping of dorsal onlay rib graft. A 25-year-old man who had revision rhinoplasty with rib cartilage developed warping of the dorsal implant $(A)$; during the revision surgery, the warped costal cartilage graft was removed, recarved, and reinserted with mastoid periosteum reinforcement over the radix $(B, C)$; frontal view 6 months after surgery shows a straightened dorsum (D)

Flat and straight pieces of cartilage were carved from his 6th costal cartilage using a No. 10 blade. After dividing the lower lateral cartilages and elevating the septal mucosa, a septal extension graft was designed from the rib cartilage to reach the anterior nasal spine inferiorly and to extend the nasal tip anteriorly while rotating it caudally. The septal extension graft was reinforced with a septal batten graft and an extended spreader graft to prevent twisting forces.

Afterwards, extended lateral crural strut grafts were employed to strengthen the lateral compartment and match the elongated central compartment [Figure 18D-F]. After completely separating the lateral crus from 

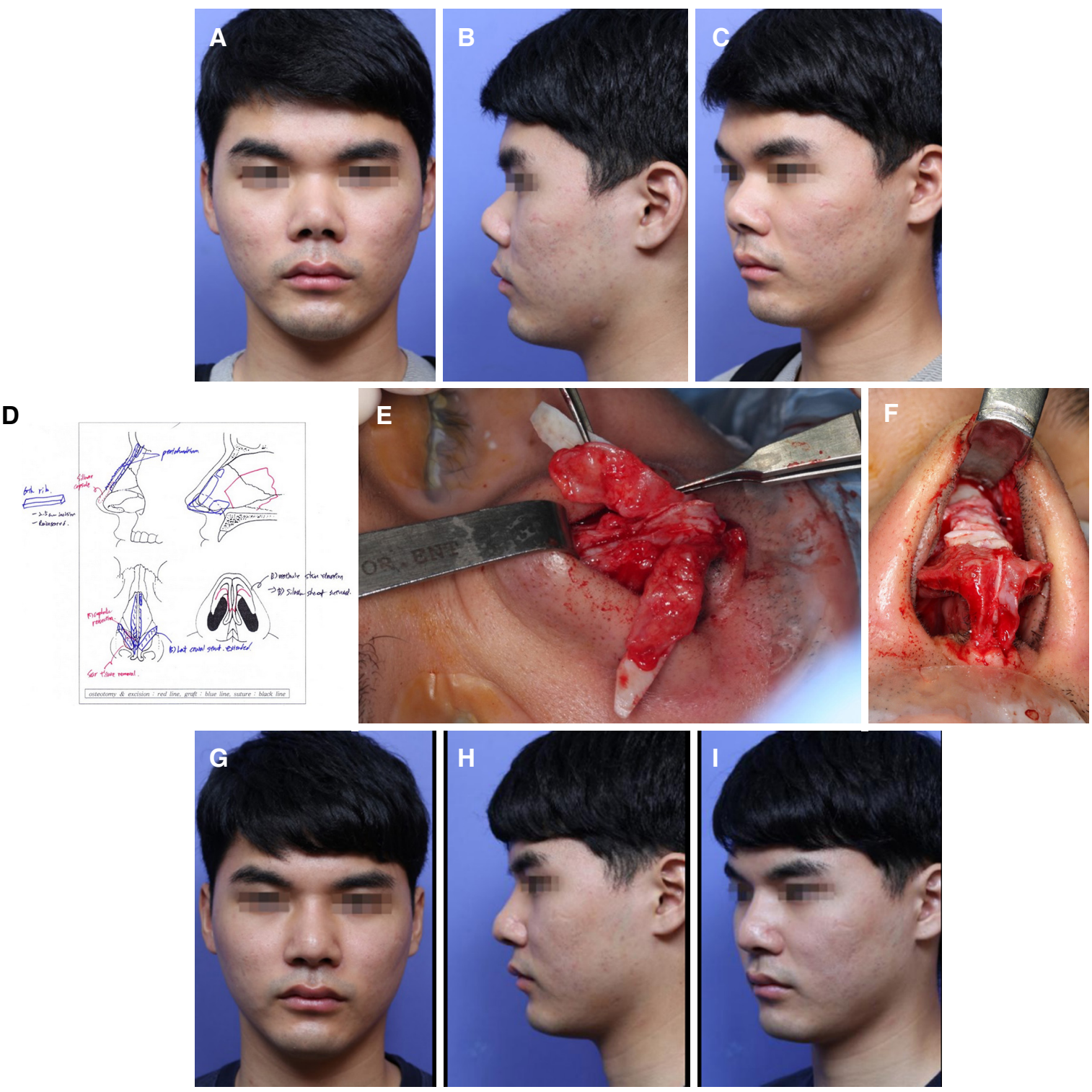

Figure 18. Correction of the short nose due to contracture after multiple rhinoplasties. A short and contracted nose, developed after infected dorsal silicone implant removal is evident from the preop photos (A, B, C); The surgical diagram shows septal extension graft, dorsal onlay graft, bilateral extended spreader grafts, lateral crural onlay grafts, shield graft and cap graft were placed using irradiated homologous costal cartilage (D); After wide release of the skin envelope, a septal extension graft is placed and reinforced with extended spreader grafts. The released lower lateral cartilages and reinforced with extended lateral crural strut grafts. Perichondrium is added for camouflage (E, F). One year after revision surgery using rib cartilage, the nose looks much better than before. His dorsum is well elevated and the tip is caudally rotated $(\mathrm{G}, \mathrm{H}, \mathrm{I})$

the vestibular mucosa, a long, straight piece of cartilage fashioned from the rib cartilage was sutured to the undersurface of the lateral crus with their cephalic ends extending to the pyriform aperture so that they held the skin tension applied on the tip and lateral crus. In this way, lateral crural strut grafts strengthen the lateral compartment and help to stabilize the dome in a more favorable position. Because the vestibular skin is dissected off the lateral crus, it helps to reposition the vestibular mucosa more caudally.

Finally, the dorsum was elevated using a dorsal graft carved from the rib. A strip of perichondrium over the costal cartilage was used as a dorsal onlay graft making a smooth transition from bony dorsum to cartilaginous dorsum. The dorsal onlay graft was extended to include the nasion to elongate the nose. 

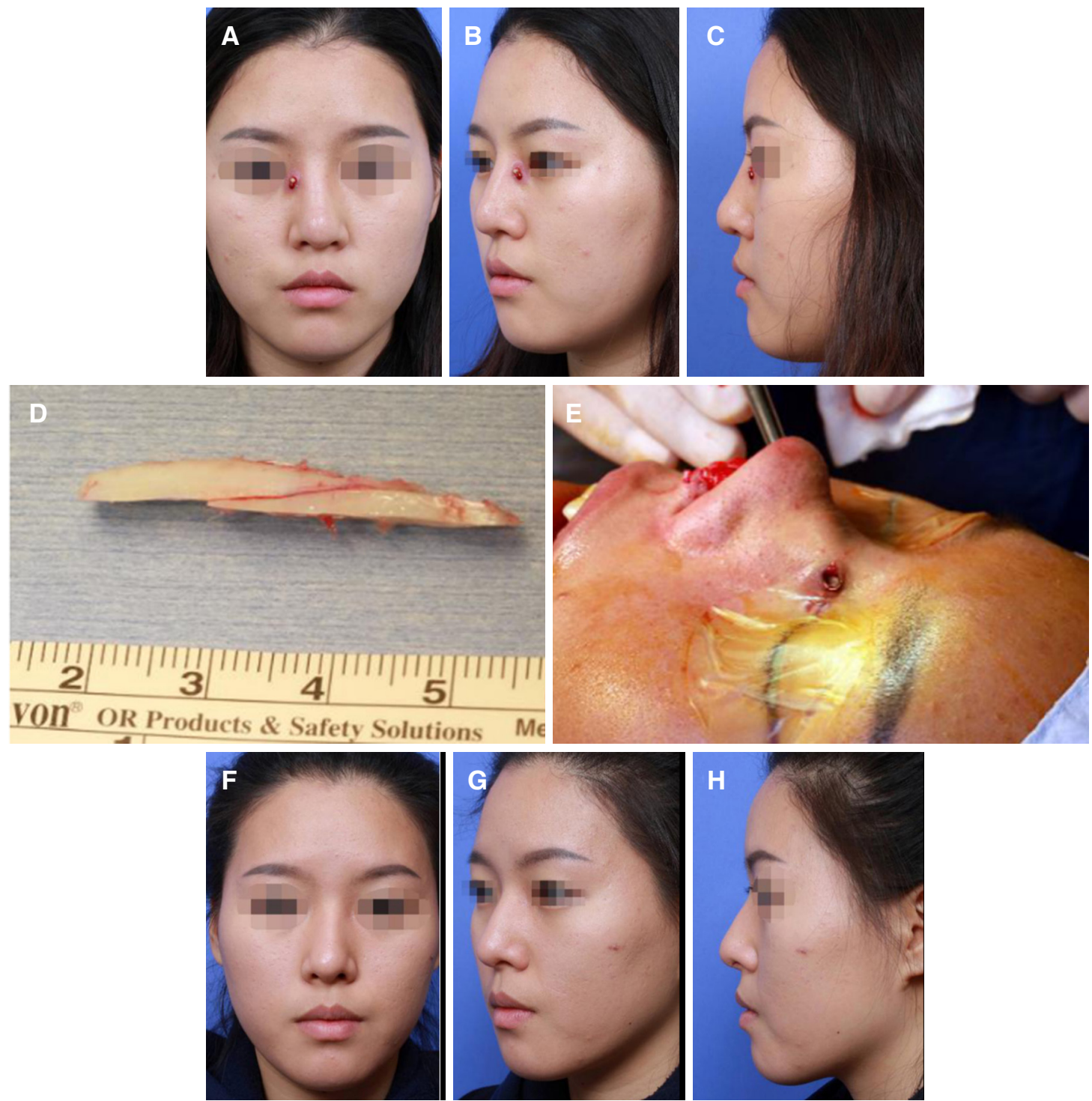

Figure 19. Case of a 19-year old female who presented with implant infection after nasal augmentation. Preoperative pictures show evident signs of infection (A, B, C). The implant was removed, and the dorsum was subsequently augmented with autogenous rib cartilage $(D, E)$ in the same setting. Tip-surgery was done using septal extension graft. One-year post-operative photos show a well restored dorsal height with appropriate tip projection and rotation of the tip $(F, G, H)$

Appearance one year after the operation shows improved nasal appearance. Caudal rotation of the nasal tip, a decreased nasolabial angle, and increased height of nasal dorsum make the nose appear significantly longer than before [Figure 18G-I].

\section{Case 2: infection after primary rhinoplasty using silicone implant. Removal and immediate reconstruction with autologous rib cartilage graft}

A 19-year-old female presented with implant infection after nasal augmentation with silicone a year before. Exudate and frank pus is noted coming from the skin of the nasal sidewall near the left medial canthus with a polly beak deformity on the lateral view [Figure 19A-C]. The implant and the surrounding granulation tissue were removed, followed by irrigation with betadine solution. The dorsum was subsequently augmented with autogenous rib cartilage [Figure 19D and E] in the same setting. Tip-surgery was done using septal extension graft. One-year post-operative photos show a well restored dorsal height with appropriate tip projection and rotation of the tip [Figure 19F-H]. 


\section{CONCLUSION}

The use of autogenous grafting material, especially the rib cartilage, is frequently needed when dealing with revision rhinoplasty cases associated with alloplast complications. Wise use of costal cartilage in revision rhinoplasty involves mastering intricacies that can decrease complications and improve results. Safe harvesting minimizing the complications, appropriate designing and carving to maximize the results and avoid warping, and appropriate postoperative care are all important. The rhinoplasty surgeon should be familiar with the common scenarios in which the rib cartilage is necessary as well as with the various technical aspects necessary to reduce complications.

\section{DECLARATIONS}

\section{Authors' contributions}

Wrote the paper: Won TB, Jin HR

\section{Availability of data and materials}

Not applicable.

\section{Financial support and sponsorship}

None.

\section{Conflicts of interest}

Both authors declared that there are no conflicts of interest.

\section{Ethical approval and consent to participate}

Not applicable.

\section{Consent for publication}

Not applicable.

\section{Copyright}

(c) The Author(s) 2019.

\section{REFERENCES}

1. Won TB, Jin HR. Revision rhinoplasty in Asians. Ann Plast Surg 2010;65:379-84.

2. Toriumi DM, Swartout B. Asian rhinoplasty. Facial Plast Surg Clin North Am 2007;15:293-307.

3. Jin HR, Won TB. Nasal hump removal in Asians. Acta Otolaryngol Supp1 2007;558:95-101.

4. Jin HR, Won TB. Nasal tip augmentation in Asians using autogenous cartilage. Otolarygol Head Neck Surg 2009;140:526-30.

5. Won TB, Jin HR. Nuances with the Asian tip. Facial Plast Surg 2012;28:187-93.

6. Jin HR, Won TB. Recent advances in Asian rhinoplasty. Auris Nasus Larynx 2011;38:157-64.

7. Jin HR, Won TB. Rhinoplasty in the Asian Patient. Clin Plast Surg 2016;43:265-79.

8. Zeng Y, Wu W, Yu H, Yang J, Chen G, et al. Silicone implant in augmentation rhinoplasty. Ann Plast Surg 2002;49:495-9.

9. Ahn JM, Honrado C, Horn C. Combined silicone and cartilage implants: augmentation rhinoplasty in Asian patients. Arch Facial Plast Surg 2004;6:120-3.

10. Deva AK, Merten S, Chang L. Silicone in nasal augmentation rhinoplasty: a decade of clinical experience. Plast Reconstr Surg 1998;102:1230-7.

11. Tham C, Lai YL, Weng CJ, Chen YR. Silicone augmentation rhinoplasty in an Oriental population. Ann Plast Surg 2005;54:1-5.

12. Endo T, Nakayama Y, Ito Y. Augmentation rhinoplasty: observations on 1,200 cases. Plast Reconstr Surg 1991;87:54-9.

13. Mendelsohn M, Dunlop G. Gore-Tex augmentation grafting in rhinoplasty-is it safe? J Otolaryngol 1998;27:337-41.

14. Rothstein SG, Jacobs JB. The use of Gore-Tex implants in nasal augmentation operations. Entechnology 1989;68:40-5.

15. Jin HR, Lee JY, Yeon JY, Rhee CS. A multicenter evaluation of the safety of Gore-Tex as an implant in Asian rhinoplasty. Am J Rhinol 2006;20:615-9.

16. Jung DH, Moon HJ, Choi SH, Lam SM. Secondary rhinoplasty of the Asian nose: correction of the contracted nose. Aesthetic Plast Surg 2004;28:1-7. 
17. Won TB, Jin HR. Immediate reconstruction with autologous cartilage after removal of infected alloplast in revision rhinoplasty. Otolaryngol Head Neck Surg 2012;147:1054-9.

18. Jin HR. Correction of the short, contracted nose. In: Jin HR, editor. Aesthetic Plastic Surgery of the East Asian Face 1st ed. New York: Thieme Medical Publishers; 2016. pp. 93-113.

19. Park JH, Mangoba DC, Mun SJ, Kim DW, Jin HR. Lengthening the short nose in asians: key maneuvers and surgical results. JAMA Facial Plast Surg 2013;15:439-47.

20. Sunwoo W, Jung H, Kim DW, Jin HR. Immunohistochemical analysis of capsular contracture in silicone implant rhinoplasty. JAMA Facial Plast Surg 2017;19:436-7.

21. Harris S, Pan Y, Peterson R, Stal S, Spira M. Cartilage warping: an experimental model. Plast Reconstr Surg 1993;92:912-5.

22. Kim DW, Shah AR, Toriumi DM. Concentric and eccentric carved costal cartilage: a comparison of warping. Arch Facial Plast Surg 2006;8:42-6. 